\title{
Erweiterung des Treuhandpartnernetzes im Kanton Bern
}

\section{Die besten Ärzte der Welt sind: Dr. Ruhe, Dr. Diät und Dr. Fröhlichkeit}

Oder was meinen Sie als Ärztin oder Arzt mit langjähriger Erfahrung? Ganz so falsch liegt Jonathan Swift (irischer Schriftsteller und Satiriker) mit seiner Aussage gegen Ende des 17. Jahrhunderts wohl nicht - oder?

\section{Ruhe gefällig?}

Sehnen Sie sich nach mehr Ruhe und möchten einfach nur die Stille geniessen? Wir haben das Rezept: Mit unserer langjährigen Erfahrung in der Steuer-, Treuhandund Finanzberatung sorgen wir dafür, dass Sie als Arzt/ Ärztin zu Ihrer wohlverdienten Ruhe kommen. Durch unsere langjährige Erfahrung sorgen wir dafür, dass Sie sich bei uns von Kopf bis Fuss wohl fühlen und von unseren individuellen und auf Ihre Bedürfnisse angepassten Dienstleistungen profitieren können. Dadurch erhalten Sie mehr Zeit für Ihre wohlverdiente Ruhe.

\section{Abnehmen ohne Diät?}

Gerne würden wir Ihnen an dieser Stelle gesunde und klinisch erprobte Diät-Tipps abgeben. Dies gehört aber leider nicht zu unserer Kernkompetenz. Jedoch können wir dafür sorgen, dass Sie mehr Zeit zur «richtigen» Ernährungs- und Lebensweise haben. Wir haben kein Rezept für das Abnehmen, aber abnehmen können wir Ihnen trotzdem etwas: das Führen Ihrer Buchhaltung und der Saläradministration sowie weitere administrative Aufgaben, damit Ihnen mehr Raum zur Erholung bleibt.

\section{Warum machen Fröhlichsein und Lachen gesund?}

Das Sprichwort «Lachen ist die beste Medizin» kommt nicht von ungefähr. Sogar wissenschaftliche Studien beweisen jetzt, was der Volksmund schon lange vermutet hat: Lachen aktiviert im menschlichen Organismus eine Vielzahl von biochemischen Prozessen, welche sich positiv auf Körper und Geist auswirken. Lachen versorgt das Hirn mit Sauerstoff, baut Stresshormone ab und unterstützt somit die Immunabwehr und setzt zu guter Letzt Glückshormone frei.

Wunder bewirken können wir leider keine. Aber wir hoffen, dass wir für einen kurzen Augenblick Ihre Lachmuskeln aktivieren konnten und Sie dadurch mehr Sauerstoff in Ihren Organen hatten, was wiederum Ihre Kondition fördert und das Herz stärkt. Und das Herz ist ja bekanntlich eines der wichtigsten Organe überhaupt. Sie beraten ja Ihre Patientinnen und Patienten mit Herz. Wir unsere Kundschaft auch! Und bei Fragen rund um Steuern, Treuhand und Finanzen fragen Sie einfach uns.

Die Lemag Treuhand+Partner AG, der neue Vertrauenspartner FMH Services (Treuhand), ist seit vielen Jahren in der Region Oberaargau beheimatet. Marc Schwertfeger als Ihr Ansprechpartner freut sich, Sie als zukünftigen Partner zu betreuen.

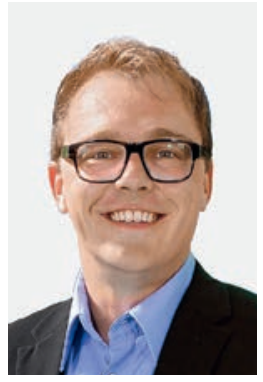

Lemag Treuhand+Partner AG

FMH Services (Treuhand)

Untere Einschlagstrasse 5

4923 Wynau

Tel. 06291810 20, Fax 0629181021

www.lemag-ag.ch

Die Lemag Treuhand+Partner AG ist ein von der FMH Services Genossenschaft empfohlenes, rechtlich und wirtschaftlich selbständiges Beratungsunternehmen.

Marc Schwertfeger

Fachmann im Finanz- und Rechnungswesen mit eidg. FA

marc.schwertfeger[at]fmhtreuhand.ch 